\title{
Poor recognition of other-race faces cannot always be explained by a lack of effort
}

\author{
Kate Crookes and Gillian Rhodes \\ ARC Centre of Excellence in Cognition and Its Disorders, School of Psychology, \\ University of Western Australia
}

Accepted version submitted to the Visual Cognition special issue on Person Perception

Date: $21^{\text {st }}$ March 2017

Word count: 7570 (including the abstract, footnotes, references, figure captions)

Keywords: face recognition, other-race effect

Contact details: $\quad$ Kate Crookes

ARC Centre of Excellence in Cognition and Its Disorders

School of Psychology

University of Western Australia

35 Stirling Highway

Crawley, Western Australia, 6009

Australia

Email: Kate.Crookes@uwa.edu.au

Gillian Rhodes

ARC Centre of Excellence in Cognition and Its Disorders

School of Psychology

University of Western Australia

35 Stirling Highway

Crawley, Western Australia, 6009

Australia

Email: Gillian.Rhodes@uwa.edu.au

Acknowledgements: We thank Stephen Pond for his assistance in programming the tasks, Elinor McKone for allowing us to use the Australian CFMT and Jia Lui for allowing us to use the Chinese CFMT. This research was supported by the Australian Research Council (ARC) Centre of Excellence in Cognition and its Disorders (CE110001021); ARC Discovery Outstanding Researcher Award to Rhodes (DP130102300). 


\begin{abstract}
People are generally better at recognising own-race than other-race faces. This "other-race effect" is very well established although the underlying causes are much debated. Social-cognitive accounts argue that the other-race effect stems from a lack of motivation to individuate other-race faces, whereas perceptual expertise accounts argue that it reflects the tuning of face-processing mechanisms by experience to own-race faces. We investigated the effort people apply to recognise own-race and other-race faces. Caucasian participants completed the Australian and Chinese Cambridge Face Memory Tasks, once with the standard timing and once with self-paced study phases. If people are less motivated to recognise other-race faces they should apply less effort, that is, when given control over viewing times they should spend less time studying other-race than own-race faces. Contrary to social-cognitive accounts there was no evidence of reduced effort for other-race faces, participants did not spend less time studying other-race than own-race faces in the self-paced condition. Moreover participants reported applying significantly more effort to telling apart other-race than own-race faces. These results are not consistent with reduced motivation to individuate other-race faces. Thus, they appear more consistent with perceptual expertise rather than social-cognitive accounts of the other-race effect.
\end{abstract}


The other-race effect - the finding that people are more accurate at recognizing faces from their own-race than faces from other-races - is a very well established phenomenon ${ }^{1}$. Original demonstrations showed that Caucasian Americans were poorer at recognizing African American than Caucasian faces (e.g., Cross, Cross, \& Daly, 1971; Malpass \& Kravitz, 1969). The effect has since been widely replicated across numerous racial groups and countries (e.g., Chiroro, Tredoux, Radaelli, \& Meissner, 2008; Ellis \& Deregowski, 1981; Michel, Rossion, Han, Chung, \& Caldara, 2006; Pauker et al., 2009; Wan, Crookes, Reynolds, Irons, \& McKone, 2015; Zhao, Hayward, \& Bülthoff, 2014).

The other-race effect is of both theoretical and practical importance. In legal contexts it can have catastrophic consequences leading to errors in eyewitness identification (Scheck, Neufeld, \& Dwyer, 2003). Less seriously, failing to recognize a friend or work colleague of another race could result in awkward social situations and potentially cause great offence similar to the experiences of people with prosopagnosia (Yardley, McDermott, Pisarski, Duchaine, \& Nakayama, 2008). Theoretically, understanding the causes of the other-race effect can help reveal the processes that support expert face recognition and person perception (e.g., Sporer, 2001; Valentine, 1991). However, despite decades of research, the underlying causes of the other-race effect are still much debated (e.g., Hugenberg, Young, Bernstein, \& Sacco, 2010; Rossion \& Michel, 2011).

There are two main broad theoretical accounts of the other-race effect: perceptual expertise accounts and social-cognitive accounts. Perceptual

\footnotetext{
${ }^{1}$ Here the term race is used, as is conventional in this literature, to refer to visually distinct groups of people.
} 
expertise theories argue that specialized face processing mechanisms become tuned through experience to operate optimally for own-race faces (e.g., G. Rhodes, Hayward, \& Winkler, 2006; Rossion \& Michel, 2011; Tanaka, Kiefer, \& Bukach, 2004; Valentine, 1991). When these mechanisms are presented with an other-race face they are not equipped to encode it as successfully as they would an own-race face. Importantly, according to perceptual expertise theories the other-race effect is not something that can be easily overcome - extensive experience is required to become an other-race face recognition expert.

In contrast, social-cognitive accounts argue that people are capable of recognizing other-race faces to the same level of accuracy as own-race faces, but that they are not motivated to do so (e.g., Hugenberg, Miller, \& Claypool, 2007; Levin, 2000; Meissner, Brigham \& Butz, 2005; Sporer, 2001). These theories assume individuation is an effortful and resource intensive process that is spontaneously applied to own-race faces given their social importance. It is argued that other-race faces are not motivating and are therefore instead processed using less effortful mechanisms. There have been several proposals regarding the exact mechanisms underlying the lack of individuation for otherrace faces including out-group categorization (e.g., Hugenberg et al., 2007), lack of attention (e.g., Levin, 2000) and use of a familiarity rather than recollection strategy (e.g., Meissner et al., 2005). More recently Hugenberg and colleagues have proposed the "Categorization Individuation Model" (Hugenberg, Wilson, See \& Young, 2013; Hugenberg et al., 2010; Young \& Hugenberg, 2012) of the other-race effect which attempts to incorporate both social and perceptual expertise factors. However, all social-cognitive accounts, including the Categorization Individuation Model, agree that the other-race effect can be 
overcome, or at least attenuated, if an individual is motivated to individuate other-race faces.

Several studies have shown that increasing participants' motivation to individuate other-race faces can reduce and even remove the other-race effect (Ackerman et al., 2006; Hehman, Mania, \& Gaertner, 2010; Hugenberg et al., 2007; Krumhuber \& Manstead, 2011; G. Rhodes, Locke, Ewing, \& Evangelista, 2009; Young, Bernstein, \& Hugenberg, 2010; Young \& Hugenberg, 2012). Manipulations have included simply instructing participants to individuate other-race faces (Hugenberg et al., 2007; G. Rhodes et al., 2009; Young et al., 2010; Young \& Hugenberg, 2012), providing cues which lead to other-race faces being classed as in-group (Hehman et al., 2010) and presenting faces with angry facial expressions (Ackerman et al., 2006; Krumhuber \& Manstead, 2011; Young \& Hugenberg, 2012). However, these results do not directly demonstrate a lack of motivation for other-race compared to own-race faces as the core driver of the other-race effect. To argue that motivation contributes to the other-race effect it is necessary to show a relative deficit in the spontaneous or default level of motivation for other- versus own-race faces. Although these previous studies show that increasing motivation to individuate other-race faces can reduce the other-race effect, the spontaneous or default level of motivation to individuate other-race faces might actually be as strong for other-race faces as for own-race faces, and these manipulations might increase it to a level greater than that for own-race faces (Rossion \& Michel, 2011).

Few studies have investigated spontaneous motivation to individuate own-race and other-race faces. A recent exception by Wan et al. (2015) showed that participants reported applying significantly more effort to the task of 
recognising other-race than own-race faces. The most strongly endorsed reason for applying greater effort to other-race faces was the realisation that other-race faces are more difficult to recognise than own-faces. Despite the extra effort participants reported applying to other-race faces a strong other-race effect was observed. These results do not support a social-cognitive, motivational account of the other-race effect, which would predict reduced effort for other-race faces.

In Wan et al.'s (2015) study it is not clear how the greater effort reported for other-race than own-race faces was manifested given that the presentation conditions were standardised across the two races of face and participants had little control over the learning conditions. Another way to investigate a potential difference in effort applied to own-race versus other-race face recognition is to give participants control over the presentation duration in the study phase of a recognition memory task. Under these conditions, social-cognitive accounts would predict that, due to a lack of motivation, participants would spend less time studying other-race than own-race faces and that this would increase the size of the other-race effect. In contrast, perceptual expertise accounts would predict that participants would spend more time studying other-race faces, given that they are aware that these are harder to recognize (Wan et al., 2015). Such meta-cognitive control is expected based on results for other stimuli (e.g., words, Tullis \& Benjamin, 2011). However, perceptual expertise accounts predict that, despite greater effort being applied to other-race faces, the size of the other-race effect will be relatively unaffected, due to the limitations set by the lack of perceptual expertise, or possibly slightly reduced, given the generally beneficial effects of increasing study time on recognition performance (e.g., Valentine \& Bruce, 1986). 
Two recent studies gave participants control over the presentation duration of faces in the study phase of a recognition memory task for own-race and other-race faces (M. G. Rhodes, Sitzman, \& Rowland, 2013; Tullis, Benjamin, \& Liu, 2014). Neither study found any difference in self-paced study times for own-race and other-race faces suggesting no difference in the spontaneous effort applied to own-race and other-race faces. Moreover, Tullis et al. (2014) found no difference in the size of the other-race effect between the self-paced condition and a fixed-rate condition. M.G. Rhodes et al. (2013) found a significant otherrace effect under self-paced study conditions but did not include a fixed-rate control condition for comparison of the size of the effect. These results are inconsistent with a social-cognitive, motivational account of the other-race effect, but consistent with a perceptual expertise account.

A problem with these two studies is that the same images were used at study and test and hair was shown (M. G. Rhodes et al., 2013; Tullis et al., 2014). It is therefore possible that participants focused on non-face cues, including pictorial, image-based cues and hair cues, to complete the task, and so their study times may not reflect their effort in remembering faces per se. Another problem is that viewing times were not directly measured, but simply assumed to match stimulus durations. Related to this issue, social-cognitive accounts would predict shorter viewing times to other-race than own-races faces even in conditions where participants do not have control over viewing times (i.e., participants may look away from other-race faces if they are less motivated to encode them). Previous studies have not been able to test this prediction.

The present study assessed the spontaneous effort applied to individuate own-race versus other-race faces, by examining both self-reported effort ratings 
and allocations of actual face viewing time at study in a recognition memory task. Improving on previous studies, hair was masked and the task required face recognition across changes in viewpoint and lighting. A social-cognitive account of the other-race effect predicts longer viewing times and greater reported effort for own-race compared to other-race faces under both self-paced and standard study-time conditions. It also predicts a larger other-race effect in the self-paced than the standard study time condition because viewing times are expected to be reduced for other-race faces in the self-paced condition. In contrast, as noted above, a perceptual expertise account predicts no such increase in the other-race effect in the self-paced condition, and greater self-reported effort for other-race than own-race faces.

We used two versions of the Cambridge Face Memory Test (Duchaine \& Nakayama, 2006) to measure the other-race effect in recognition memory: the Australian CFMT (McKone et al., 2011) and the Chinese CFMT (McKone et al., 2012). These tests require participants to learn six individuals and then recognize them across changes in lighting and viewpoint. We tested only Caucasian participants, but given that the Australian and Chinese versions of the CFMT produce a reliable other-race effect in both Caucasian and Asian participants (e.g., Wan et al., 2015) we can be confident that any other-race effect is not simply a stimulus effect. Participants completed both CFMTs once with standard study times and once with self-paced study times, in counterbalanced order. In an important improvement over previous studies, we used an eyetracker to measure the actual time spent viewing the faces during the study phases in both the standard and self-paced viewing time conditions. After participants had completed the four CFMTs they were asked to rate the effort 
they had put into telling apart both Caucasian and Asian faces. To ensure that they met our inclusion criteria (i.e., 100\% Caucasian ancestry and no more than 2 years living in a predominantly Asian country) participants also completed a racial background questionnaire. Finally, to characterize the degree of other-race contact in our sample, participants completed the Hancock and Rhodes (2008) contact questionnaire.

\section{Method}

\section{Participants}

Participants were 38 students (28 female; Age: Mean = 18.7 years, $S D=$ 2.0 , range $=17-27$ years) at the University of Western Australia who received course credit for their participation in the 1.5 hour experiment. All were Caucasian and reported only Caucasian ancestry. None reported ever having lived in a majority Asian country. All reported normal or corrected-to-normal vision. Participants wore glasses $(n=4)$ or contact lenses $(n=2)$ if they normally would when working at a computer. Seven additional participants were excluded due to reporting non-Caucasian ancestry $(\mathrm{n}=3)$, living in a predominantly Asian country for more than 2 years $(n=2)$, computer failure $(n=1)$ or failure to complete the task in the time allowed $(n=1)$. As expected of a Caucasian Australian sample, on a scale where a higher score indicates a greater degree of contact (maximum score $=6$ ) participants reported significantly more contact with Caucasian $(M=5.3, S D=0.5)$ than $A \operatorname{sian}(M=3.7, S D=0.7)$ people, $t(37)=$ $9.69, p<.001$, Cohen's $d=1.57$. 


\section{Materials and procedure}

General Procedure. All tasks were presented on a 21.5" iMac computer. Responses were entered by the participant using a standard keyboard. Participants first completed the CFMTs followed by the questionnaires.

CFMTs. Each participant completed four CFMTs: Asian standard-timing, Caucasian standard-timing, Asian self-paced and Caucasian self-paced. The Caucasian version was the CFMT-Australian (McKone et al., 2011) and the Asian version was the CFMT-Chinese (McKone et al., 2012). The standard-timing versions followed the normal CFMT procedure (see Duchaine \& Nakayama, 2006 for a full description) with some minor modifications to allow for eye tracking (see below). In brief, the CFMT consists of a learning phase, a novel phase and a noise phase (see Figure 1). In the learning phase participants learn a target face in 3 different views, each presented for 3 seconds, followed by three test trials where each learned view of that target must be identified from two simultaneously presented distractors. Test faces are presented until response. This learning-test procedure is repeated for the 6 target faces resulting in 18 trials. In the novel phase, on each trial a new image (e.g., different viewpoint and/or lighting conditions) of a target is presented with two distractors until response. There are 5 trials per target (i.e., 30 trials in total) with trials from the different targets intermixed across the novel phase. In the noise phase more new images of the targets are presented this time overlaid with visual noise. Again, on 
each trial the target is presented with two distractors until response. There are 4 trials per target (24 in total) intermixed across the phase. Before both the novel and the noise phases participants are presented with a review screen depicting all 6 targets for 20 seconds. The faces are all male and hair and clothing are masked.
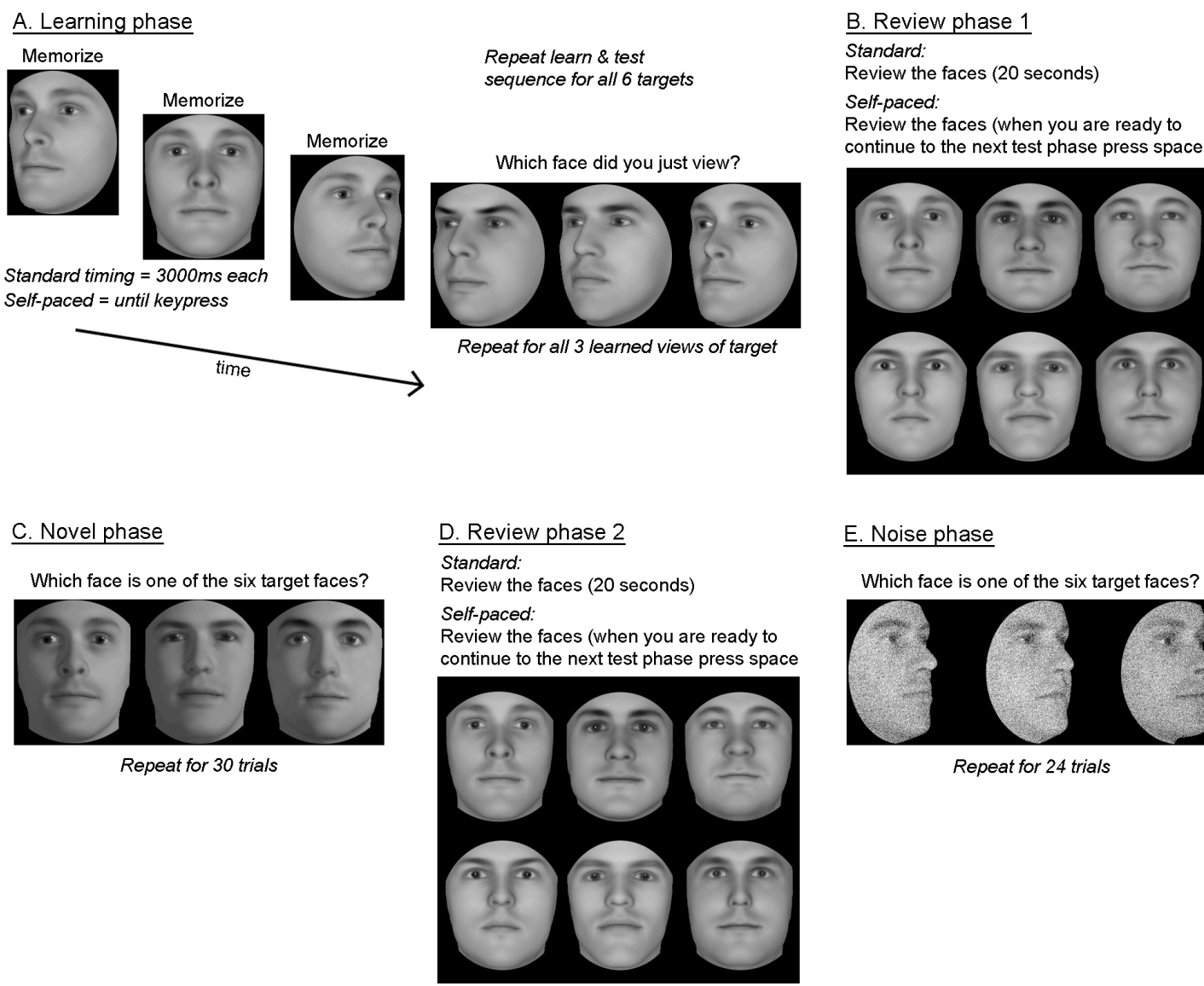

\section{E. Noise phase}

Which face is one of the six target faces?

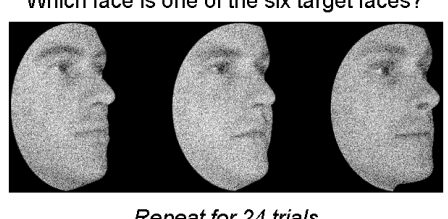

Repeat for 30 trials

Repeat for 24 trials

Figure 1. CFMT procedure. Note that the example faces depicted here were

randomly generated using FaceGen Modeller 3.5.3 and do not appear in the tests which used photographic stimuli.

The self-paced condition differed from the standard-timing condition only in the length of presentation in the learning phase and the review screens. In the 
learning phase participants were told to study the faces for as long as they liked and to press the spacebar when they were ready to move on to the next image. Similarly before the review screens they were told they had some time to review the faces and to press the spacebar when they were ready to move on to the next test phase.

All the CFMTs were programmed using SR Research Experiment Builder. Some modifications to the standard CFMT procedure were required to accommodate the eye tracker. First, a 9-point calibration routine was performed before the practice phase, learning phase, review screens, novel phase and noise phase. Second, prior to each trial a fixation point was presented at the top centre of the screen. This position was chosen so as to not overlap with the subsequent stimulus requiring an eye-movement to the stimulus once it appeared. The trial was initiated by the experimenter when the participant was looking at the fixation point.

Four order conditions were used: 1) Asian standard-timing, Caucasian standard-timing, Asian self-paced, Caucasian self-paced; 2) Caucasian standardtiming, Asian standard-timing, Caucasian self-paced, Asian self-paced; 3) Asian self-paced, Caucasian self-paced, Asian standard-timing, Caucasian standardtiming; 4) Caucasian self-paced, Asian self-paced, Caucasian standard-timing, Asian standard-timing.

During the CFMTs a desk-mounted SR Research Eyelink 1000 eye tracker was used to record eye movements using the default settings. A viewing distance of $52 \mathrm{~cm}$ was maintained using a chin rest. For each participant the dominant eye was tracked (8 left; 30 right). For analysis, each face was defined as a single, oval-shaped area of interest. 
Questionnaires. Following completion of the four CFMTs participants completed an effort rating task, racial background questionnaire and own-race and other-race contact questionnaire in that order. Questionnaires were presented on the computer and were programmed using Psyscope $\mathrm{X}$ (psy.ck.sissa.it; Cohen, MacWhinney, Flatt, \& Provost, 1993).

Self-rated effort on the CFMTs was assessed using the ratings task developed by Wan et al. (2015). Participants were asked to rate the effort they put into telling apart each race of face on a 7-point scale where $1=$ "just normal effort, nothing special" and 7 = "a lot of special effort". Participants were first asked, "On the memory task, how much special effort did you put into telling apart the faces of the Caucasian people you saw?" they were then asked, "On the memory task, how much special effort did you put into telling apart the faces of the Asian people you saw?". Following these ratings participants were asked the following open-ended question, "Did you put more effort into one group of faces than the other? Please give the reason behind your answer".

Participants were then asked to report their race and that of their parents, their country of birth, country/region of ancestry, if they had lived in a country other than Australia (and if so for how long), and if they were an overseas student when did they move to Australia. This information was used to exclude individuals who reported non-Caucasian ancestry and/or having lived in a predominantly Asian country. 
Self-rated contact with own-race and other-race people was assessed using a modified ${ }^{2}$ version of the Hancock and Rhodes (2008) questionnaire. Participants rated their agreement with 14 statements on a six-point scale $(1=$ very strongly disagree, 6 = very strongly agree). Half the statements were about their contact with Caucasian people and half were about their contact with Asian people. Contact with own-race and other-race people was calculated as the mean rating for the 7 statements regarding each race. A higher score indicates a greater level of contact.

\section{Results}

Face viewing time during study phases

Eye-tracking was used to calculate the time that each participant spent viewing faces during the three study phases (learning, first review, second review), for each race of face and study-time condition. In the learning phase three different viewpoints of each of the six identities were presented individually. The median viewing time was calculated across all 18 items. In the review phases before each of the novel and the noise test phases all six faces were presented on the screen at once. In the review phases total viewing time across all six faces was calculated for each participant. The means of these study time measures for own-race and other-race faces are presented in Figure 2. Not surprisingly, there were large differences in variability between the standard and the self-paced study-time conditions, as can be seen in the error bars in

2 The word "Chinese" was replaced with "Asian" to reflect the likelihood that participants may not be aware of the exact ethnic origin of the people they interact with. 
Figure 2. The two study-time conditions were therefore analysed separately. As the learning and review phases had different viewing time measures they were also analysed separately. For the learning phases 2-way mixed model analyses of variance (ANOVA) were performed on the mean median viewing times with face race (own-race, other-race) as a within participant variable and study-time condition order (standard first, self-paced first) as a between participants variable. For the review phases, 3-way mixed model ANOVAs were performed on the mean viewing times with review phase (first review, second review) and face race (own-race, other-race) as within participant variables and study-time condition order (standard first, self-paced first) as a between participants variable.
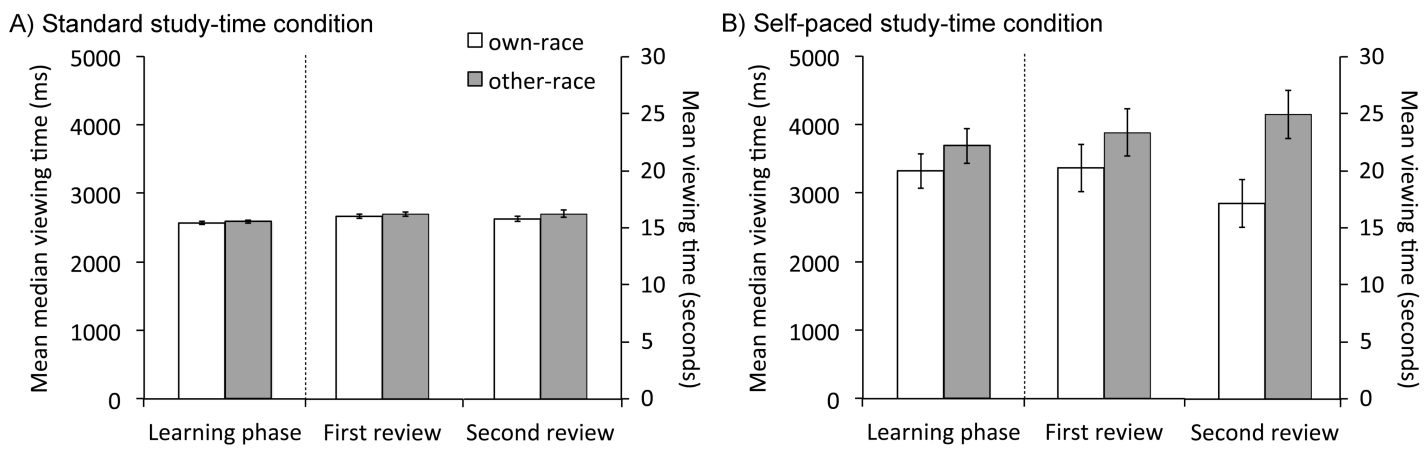

Figure 2. Mean time spent viewing own-race and other-race faces during the study phases of the CFMT with A) standard presentation times and B) self-paced presentation times. Note the different scales for the learning and review phases. In the learning phase the bars show the mean of the participants median viewing times for the 18 items in that phase. In the review phases the bars show the mean time spent viewing the 6 faces on the review screens. Standard error bars are appropriate for within-participants comparison of own-race vs. other-race faces (i.e., $\pm 1 S E M$ of the own-race - other-race difference score). 
Standard study-time condition. In the standard study-time condition there was no indication of any difference in viewing time for own-race versus otherrace faces in any condition - participants looked at the faces for the time they were presented on the screen. For the learning phase (Figure 2A) there were no significant main effects or interactions, all $F \mathrm{~s}<1$, all $p \mathrm{~s}>.32$, all $\eta_{\mathrm{p}}{ }^{2}<.028$. For the review phases (Figure 2A), there was a theoretically unimportant interaction between review phase and study-time condition order, $F(1,36)=10.94, M S E=$ $7.97 \times 10^{5}, p=.002, \eta_{\mathrm{p}}^{2}=.23$, which was not followed up. No other main effects or interactions were significant all $F \mathrm{~s}<3.17$, all $p \mathrm{~s}>.09$, all $\eta_{\mathrm{p}}{ }^{2}<.09$.

Self-paced study-time condition. Contrary to the predictions of a socialcognitive account, viewing times in the review phases were longer, not shorter, for other-race than own-race faces (Figure 2B). There was a significant main effect of face race, $F(1,36)=12.38, M S E=8.8 \times 10^{7}, p=.001, \eta_{\mathrm{p}}^{2}=.26$, resulting from longer viewing times to other-race $(M=24.1 \mathrm{sec}, S D=13.9)$ than own-race faces $(M=18.7 \mathrm{sec}, S D=11.0)$. Again, there was a theoretically unimportant interaction between review phase and study-time condition order, $F(1,36)=$ $7.43, M S E=5.12 \times 10^{7}, p=.01, \eta_{\mathrm{p}}^{2}=.17$, which was not followed up ${ }^{3}$. No other main effects or interactions were significant, all $F \mathrm{~s}<2.86$, all $p \mathrm{~s}>.10$, all $\eta_{\mathrm{p}}{ }^{2}<.08$.

\footnotetext{
${ }^{3}$ Analysis of participants who completed the self-paced block first revealed a similar pattern to that of the full analysis, that is, a tendency toward longer viewing times for other-race than own-race faces (see Supplementary Materials for analysis). Importantly there was no evidence that other-race viewing times were shorter than own-race viewing times as would be predicted by socialcognitive accounts of the other-race effect.
} 
There were no significant main effects or interactions in the learning phase, all Fs $<2.31$, all $p s>.13$, all $\eta_{\mathrm{p}}{ }^{2}<.07$.

Viewing time for other-race faces. The differences in viewing times for other-race faces in the self-paced condition compared to the standard study-time condition were in the opposite direction to that predicted by a social-cognitive account of the other-race effect. As shown in Figure 2 viewing times for both own-race and other-race faces increased in the self-paced compared to the standard study-time condition. To explore whether this increase in study times was larger for other-race than own-race faces we calculated a self-paced minus standard viewing time difference score. For the review phases an ANOVA on the difference score with face race (own-race, other-race) and review phase (first review, second review) as within participant variables and study-time condition order (standard first, self-paced first) as a between participants variable revealed a significant main effect of face race, $F(1,36)=9.77, M S E=9.76 \times 10^{7}, p$ $=.004, \eta_{\mathrm{p}}{ }^{2}=.21$, indicating that viewing times in the self-paced condition increased more relative to the standard condition for other-race $(M=7878 \mathrm{~ms}$, $S D=12493)$ than own-race faces $(M=2782 \mathrm{~ms}, S D=9363)$. There was a theoretically irrelevant significant interaction between review phase and studytime condition order, $F(1,36)=10.77, M S E=4.68 \times 10^{7}, p=.002, \eta_{\mathrm{p}}^{2}=.23$, which was not followed up. No other main effects or interactions were significant, all $F \mathrm{~s}<2.48$, all $p s>.13$, all $\eta_{\mathrm{p}}^{2}<.07$.

An ANOVA for the learning phase with face race (own-race, other-race) as a within participants variable and study-time condition order (standard first, self-paced first) as a between participants variable produced no significant main 
effects or interactions, all $F \mathrm{~s}<2.60$, all $p \mathrm{~s}>.31$, all $\eta_{\mathrm{p}}{ }^{2}<.07$.

\section{Recognition}

Figure 3 shows recognition accuracy (\% correct) in the novel phase of the CFMT $^{4}$. There was a strong other-race effect in both the standard, $t(37)=6.43, p$ $<.001$, Cohen's $d=1.04$, and self-paced, $t(37)=7.66, p<.001$, Cohen's $d=1.24$, conditions. Importantly, contrary to the prediction of a social-cognitive account, giving participants control over study time did not increase the size of the otherrace effect (Figure 3). A mixed model 2-way ANOVA performed on other-race effect scores (\% correct own-race minus \% correct other-race), with study-time condition (standard, self-paced) as a within-participant variable and study-time condition order (standard-first, self-paced-first) as a between participants variable, showed no main effect of study-time condition, $F(1,36)=1.84, p=.18$, $\eta_{\mathrm{p}}{ }^{2}=.05$. There was no significant main effect of study-time condition order, $F$ $(1,36)<1, p=.63, \eta_{\mathrm{p}}^{2}=.01$, and no interaction, $F(1,36)<1, p=.34, \eta_{\mathrm{p}}^{2}=.03$

\section{Effort ratings for own-race and other-race faces}

Participants reported applying significantly greater effort to other-race $(M=5.4, S D=1.4)$ than own-race faces $(M=4.1, S D=1.8), t(37)=6.10, p<.001$, Cohen's $d=1.00$. At the individual level, 24 participants reported applying more

\footnotetext{
${ }^{4}$ Note the novel phase, which requires recognition of learned identities across changes in viewpoint or lighting, produced a strong other-race effect whereas the other phases did not (see Table S1). As expected, performance in the learning phase was at ceiling for own-race and other-race faces. Comparison of previous studies testing own-race samples revealed that the noise phases of the CFMTAustralian and CFMT-Chinese are not comparable, with the noise condition in the CFMT-Chinese much easier than in the CFMT-Australian (McKone et al., 2011; McKone et al., 2012). The other-race effect for Caucasian participants in this condition may therefore be obscured by the overall differences in difficulty.
} 
effort to other-race than own-race faces, and 14 reported applying equal effort to own-race and other-race faces. Importantly no participants reported applying more effort to own-race than other-race faces. These results do not support a social-cognitive account, which predicts reduced motivation to individuate other-race faces.

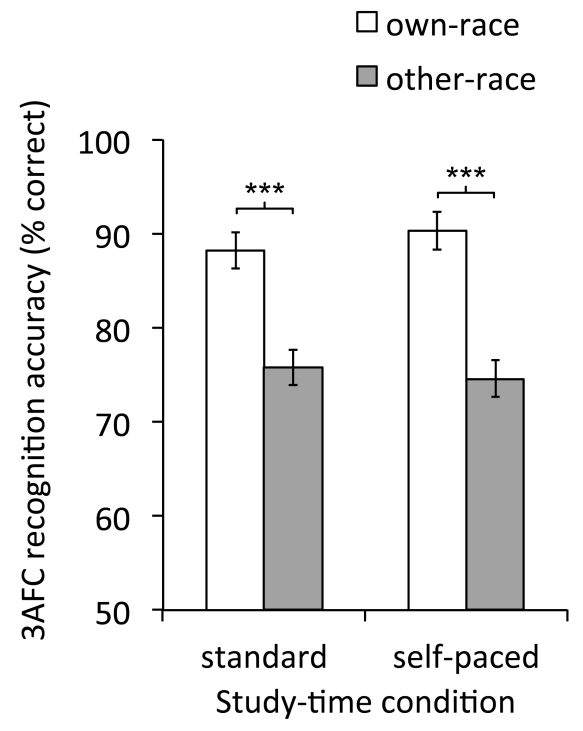

Figure 3. Recognition accuracy for own-race and other-race faces in the novel phase of the CFMT for the standard and self-paced study conditions. Chance is 33\%. Standard error bars are appropriate for within-participants comparison of own-race vs. other-race faces (i.e., \pm 1 SEM of the own-race - other-race difference score). ${ }^{* * *}=p<.001$.

To explore why participants reported applying more effort to other-race than own-race faces we looked at their responses to the open-ended question, "Did you put more effort into one group of faces than the other? Please give the reason behind your answer". Responses fell into three categories: 11 
participants indicated that Asian faces were more difficult to tell apart, 7 said that they were unfamiliar with Asian faces and 3 said Asian faces were more similar than Caucasian faces ( 2 participants failed to provide a response and 1 response was unclassifiable; for full responses see Table S2). These responses are all consistent with a perceptual expertise interpretation of the other-race effect.

\section{Discussion}

Our results provide no support for a social-cognitive account of the otherrace effect. A core claim of social-cognitive accounts is that people are less motivated to individuate other-race than own-race faces. Reduced motivation should result in reduced effort being applied to recognizing other-race compared to own-race faces. However, neither the viewing time results nor the effort ratings support this prediction. Participants did not spend more time studying own-race than other-race faces in either the standard or the self-paced studytime condition. Rather, in the self-paced condition they looked longer at otherrace than own-race faces during the review phases. In addition, participants reported applying greater effort to individuating other-race than own-race faces. On a social-cognitive account we should also see a larger other-race effect when participants have control over the viewing times, because they would be expected to reduce viewing times for other-race faces. Contrary to this prediction we found no difference in the size of the other-race effect between the self-paced and the standard study time condition. Furthermore, in the review phase there was a larger increase in viewing times between the standard and the self-paced conditions for other-race than own-race faces. 
Our results are instead consistent with perceptual expertise accounts of the other-race effect, which argue that the other-race effect can only be overcome with many years of experience. Here we found participants spontaneously put greater effort into individuating other-race than own-race faces and yet they showed a strong other-race effect that was not reduced even when they were given control over their study durations.

Our results are consistent with those of three other recent studies that examined effort applied to own-race and other-race faces (M. G. Rhodes et al., 2013; Tullis et al., 2014; Wan et al., 2015). First, we replicated the finding of Wan et al. (2015) that participants report applying more effort to other-race than own-race faces and yet showed a strong other-race effect. Such self-report measures can be problematic. It could be that rather than rating their effort the participants were actually rating the difficulty of the task. However our finding that participants increased their viewing time for other-race compared to ownrace faces in the self-paced condition suggests they did actually try harder with other-race faces. Converging evidence for this interpretation comes from a pupillometry study that found other-race faces require greater effort at encoding (Goldinger, He \& Papesh, 2009). Together, these results argue strongly that the other-race effect is not due to a lack of motivation for other-race faces.

Second, our viewing time results broadly agree with those of M.G. Rhodes et al. (2013) and Tullis et al. (2014). Both previous studies found no difference in self-paced study times for own-race versus other-race faces. Here we also found no difference in self-paced study times in the learning phase of the experiment (i.e., the first time participants were asked to study the faces). However, we also found, for the first time, evidence of longer self-paced study times to other-race 
than own-race faces but only in the review phases of the experiment. The review phases were when the participants had the chance to study the faces again having already been tested. The preceding test phases perhaps gave the participants important feedback as to the difficulty of the task for other-race faces triggering longer viewing times at review. The previous studies did not have an equivalent phase. Further, in the present study, using the eye tracker meant we employed a more accurate measure of actual viewing time than the previous studies that relied on stimulus duration.

Here, as in the previous two studies (M.G. Rhodes et al., 2013; Tullis et al., 2014), we have taken time spent studying the faces as a measure of effort expended to encode those faces. Is it possible, however, that longer viewing times for other-race faces instead reflect demand characteristics of the task? For example, participants might have looked at other-race faces for as long as or longer than own-race faces without attempting to encode them, simply to avoid appearing prejudiced. In this case, however, we would not expect them to explore the other-race faces as thoroughly as own-race faces (i.e., they would stare blankly at the screen). Analysis of the number of fixations (see the Supplementary Materials) did not support this account. Instead, participants in the review phases of the self-paced condition participants made more fixations to other-race than own-race faces, in line with the greater subjective difficulty reported for those faces. Therefore, we suggest that demand characteristics do not provide a plausible interpretation of our results.

Finally, as in Tullis et al. (2014) we found no difference in the size of the other-race effect between self-paced and fixed rate study-time conditions. Numerically the other-race effect was slightly larger in the self-paced than the 
standard timing condition raising the possibility that with greater power we might have detected a significant difference. However the increase in the otherrace effect appears to stem more from improved own-race recognition rather than poorer other-race recognition, which goes against the predictions of a social-cognitive account. Together the findings from the viewing times, effort ratings and other-race effect on recognition memory provide no evidence of reduced effort for other-race compared to own-race faces. These results are not consistent with a motivational explanation of the other-race effect and are instead consistent with perceptual expertise explanations.

We tested only Caucasian participants, which could be considered a limitation. Importantly, however, the tasks we used produce clear other-race effects in both Caucasian and Asian participants (e.g., Wan et al., 2015), so we are confident that our other-race effect cannot be a stimulus effect (i.e., the result of the Asian faces used being intrinsically more difficult to recognize than the Caucasian faces). Testing only one race of participants with only one other race of faces does, however, limit the generalizability of our conclusions regarding motivation to other races of participant or races of face. It remains to be seen whether the same results would be found with Asian participants, or indeed with other Caucasian samples (see discussion below regarding different results for Caucasian Americans viewing African American faces and Caucasians viewing Asian faces). Nevertheless, failure to demonstrate an effect of motivation in even one race of participants shows that social-cognitive accounts cannot be a universal explanation of the other-race effect. 
The role of motivation in the other-race effect has also been questioned by a growing number of studies that have failed to replicate earlier findings that increasing motivation to individuate other-race faces improves other-race face recognition and reduces the other-race effect. As reviewed in the introduction, these motivation manipulations have included encouraging categorization of other-race faces as in-group faces (Hehman et al., 2010), instructing participants to try harder to individuate other-race faces (Hugenberg et al., 2007; G. Rhodes et al., 2009; Young et al., 2010; Young \& Hugenberg, 2012) and presenting faces with angry facial expressions (Ackerman et al., 2006; Krumhuber \& Manstead, 2011; Young \& Hugenberg, 2012). Recent studies have failed to find any effect of in-group manipulation (Kloth, Shields, \& Rhodes, 2014) or individuation instructions (Tullis et al., 2014; Wan et al., 2015) on the size of the other-race effect. In a reversal of previous findings Gwinn, Barden and Judd (2015) found a larger other-race effect in Caucasian participants for faces with angry than neutral facial expressions. All these studies fail to support the idea that a lack of motivation to individuate other-race faces is a key component of the other-race effect. They are instead consistent with perceptual expertise accounts which argue that other-race face processing deficits cannot be readily overcome by increased motivation.

Another demonstration of a perceptual rather than motivational basis of the other-race effect comes from Hayward, Favelle, Oxner, Chu and Lam (in press). This study took a novel approach requiring participants to learn to name own-race and other-race faces to a criterion. They argued that this task provides intrinsic motivation to individuate all faces because greater accuracy results in finishing the task sooner. Despite this added motivation, other-race faces took 
longer to learn than own-race faces, and there was an other-race effect in recognition of new images of learned identities. Again these results suggest that other-race faces are more difficult to learn and recognize even when people are strongly motivated to succeed.

It may be important that the original evidence that increased motivation to individuate other-race faces can eliminate the other-race effect was typically obtained by testing Caucasian American participants on Caucasian and AfricanAmerican faces. In contrast, most of the recent studies that failed to find such motivation effects tested Caucasian and Asian participants looking at Caucasian and Asian faces ${ }^{5}$. This consistent discrepancy suggests that the underlying causes of the other-race effect could be different for different cultural groups, perhaps dependent on levels of experience and might be influenced by other factors such as differences in social status between groups (see Wan et al., 2015). It also raises the possibility that the causes of the other-race effect could vary within an individual depending on the race of face. For example a Caucasian American might have a "motivational other-race effect" for African American faces, a group for which they might have substantial experience, but a "perceptual other-race effect" for Asian faces, a group for which they might have relatively little experience. Future studies could explore this intriguing possibility by investigating the effects of motivation on recognition of more than one other race of face.

\footnotetext{
5 The one study that failed to find effects of motivation in Caucasian and African American participants demonstrated that the previous positive findings were likely due to problems with the stimuli and failure to properly counterbalance faces across expression conditions rather than increased motivation (Gwinn et al., 2015).
} 
Recent theories of the other-race effect such as Hugenberg et al.'s (2010) Categorization Individuation Model and Wan et al.'s (2015) Dual-Route approach have incorporated both motivation and expertise as contributing factors to the other-race effect. These theories acknowledge that there may not be one universal cause of the other-race effect and that experience and motivation may interact. However the two theories differ in that the Dual-Route approach argues there are some contexts in which motivation seems to play no role in the otherrace effect, whereas the Categorization Individuation Model argues that motivation is always a key component of the other-race effect. Our results are therefore more consistent with the Dual-Route approach (Wan et al., 2015).

\section{Conclusion}

In our increasingly globalized world recognition of other-race faces is becoming ever more important. Unfortunately simply trying hard to recognize the faces of people from other races does not seem likely to lead to accurate other-race recognition. Rather, it seems important to acknowledge that there may be limitations to performance that can arise from a lack of perceptual experience with other-race faces. 
Ackerman, J. M., Shapiro, J. R., Neuberg, S. L., Kenrick, D. T., Becker, D. V., Griskevicius, V., ... Schaller, M. (2006). They all look the same to me (unless they're angry): from out-group homogeneity to out-group heterogeneity. Psychological Science, 17(10), 836-840. doi: 10.1111/j.1467-9280.2006.01790.x

Chiroro, P. M., Tredoux, C. G., Radaelli, S., \& Meissner, C. A. (2008). Recognizing faces across continents: The effect of within-race variations on the ownrace bias in face recognition. Psychonomic Bulletin and Review, 15(6), 1089-1092.

Cohen, J., MacWhinney, B., Flatt, M., \& Provost, P. (1993). PsyScope: An interactive graphic system for designing and controlling experiments in the psychology laboratory using Macintosh computers. Behavior Research Methods, Instruments and Computers, 25, 257-271.

Cross, J. F., Cross, J., \& Daly, J. (1971). Sex, race, age, and beauty as factors in recognition of faces. Perception and Psychophysics, 10(6), 393-396.

Duchaine, B. C., \& Nakayama, K. (2006). The Cambridge face memory test: Results for neurologically intact individuals and an investigation of its validity using inverted face stimuli and prosopagnosic participants. Neuropsychologia, 44, 576-585.

Ellis, H. D., \& Deregowski, J. B. (1981). Within-race and between-race recognition of transformed and untransformed faces. American Journal of Psychology, 94(1), 27-35. doi: 10.2307/1422341 
Goldinger, S. D., He, Y., \& Papesh, M. H. (2009). Deficits in cross-race face learning: Insights from eye movements and pupillometry. Journal of Experimental Psychology: Learning, Memory and Cognition, 35(5), 11051122.

Gwinn, J. D., Barden, J., \& Judd, C. M. (2015). Face recognition in the presence of angry expressions: A target-race effect rather than a cross-race effect. Journal of Experimental Social Psychology, 58, 1-10. doi: 10.1016/j.jesp.2014.12.001

Hancock, K. J., \& Rhodes, G. (2008). Contact, configural coding and the other-race effect in face recognition. British Journal of Psychology, 99, 45-56.

Hayward, W. G., Favelle, S. K., Oxner, M., Chu, M. H., \& Lam, S. M. (in press). The other-race effect in face learning: Using naturalistic images to investigate face ethnicity effects in a learning paradigm. The Quarterly Journal of Experimental Psychology. doi: 10.1080/17470218.2016.1146781

Hehman, E., Mania, E. W., \& Gaertner, S. L. (2010). Where the division lies: Common ingroup identity moderates the cross-race facial-recognition effect. Journal of Experimental Social Psychology, 46(2), 445-448. doi: 10.1016/j.jesp.2009.11.008

Hugenberg, K., Miller, J., \& Claypool, H. M. (2007). Categorization and individuation in the cross-race recognition deficit: Toward a solution to an insidious problem. Journal of Experimental Social Psychology, 43, 334340.

Hugenberg, K., Young, S. G., Bernstein, M. J., \& Sacco, D. F. (2010). The categorization-individuation model: An integrative account of the otherrace recognition deficit. Psychological Review, 117(4), 1168-1187. 
Kloth, N., Shields, S., \& Rhodes, G. (2014). On the other side of the fence: Effects of social categorization and spatial grouping on memory and attention for own-race and other-race faces. PLoS One, 9(9), e105979. doi: 10.1371/journal.pone.0105979

Krumhuber, E. G., \& Manstead, A. S. R. (2011). When memory is better for outgroup faces: On negative emotions and gender roles. Journal of Nonverbal Behavior, 35, 51-61.

Levin, D. T. (2000). Race as a visual feature: Using visual search and perceptual discrimination tasks to understand face categories and the cross-race recognition deficit. Journal of Experimental Psychology: General, 129(4), $559-574$.

Malpass, R. S., \& Kravitz, J. (1969). Recognition for faces of own and other race. Journal of Personality and Social Psychology, 13(4), 330-334.

McKone, E., Hall, A., Pidcock, M., Palermo, R., Wilkinson, R. B., Rivolta, D., . . O'Connor, K. (2011). Face ethnicity and measurement reliability affect face recognition performance in developmental prosopagnosia: Evidence from the Cambridge Face Memory Test - Australian. Cognitive Neuropsychology, 28(2), 109-146.

McKone, E., Stokes, S., Liu, J., Cohan, S., Fiorentini, C., Pidcock, M., ... Pelleg, M. (2012). A robust method of measuring other-race and other-ethnicity effects: The Cambridge Face Memory Test format. PLoS One, 7(10), e47956. doi: 10.1371/journal.pone.0047956

Meissner, C. A., Brigham, J. C., \& Butz, D. A. (2005). Memory for own- and otherrace faces: A dual-process approach. Applied Cognitive Psychology, 19, 545-567. 
Michel, C., Rossion, B., Han, J., Chung, C.-S., \& Caldara, R. (2006). Holistic processing is finely tuned for face of one's own race. Psychological Science, 17(7), 608-615.

Pauker, K., Weisbuch, M., Ambady, N., Sommers, S. R., Adams, R. B., \& Ivcevic, Z. (2009). Not so black and white: Memory for ambigurous group members. Journal of Personality and Social Psychology, 96(4), 795-810.

Rhodes, G., Hayward, W. G., \& Winkler, C. (2006). Expert face coding: Configural and component coding of own-race and other-race faces. Psychonomic Bulletin and Review, 13(3), 499-505.

Rhodes, G., Locke, V., Ewing, L., \& Evangelista, E. (2009). Race coding and the other-race effect in face recognition. Perception, 38, 232-241.

Rhodes, M. G., Sitzman, D. M., \& Rowland, C. A. (2013). Monitoring and control of learning own - race and other - race faces. Applied Cognitive Psychology, 27(5), 553-563. doi: 10.1002/acp.2948

Rossion, B., \& Michel, C. (2011). An experience-based holistic account of the other-race face effect. In A. J. Calder, G. Rhodes, M. H. Johnson \& J. V. Haxby (Eds.), Oxford Handbook of Face Perception (pp. 215-243). Oxford: Oxford University Press.

Scheck, B., Neufeld, P., \& Dwyer, J. (2003). Actual innocence: When justice goes wrong and how to make it right (New ed.). New York: New American Library.

Sporer, S.L. (2001). Recognizing faces of other ethnic groups: An integration of theories. Psychology, Public Policy, and Law, 7(1), 36-97. doi: $10.1037 / / 1076-8971.7 .1 .36$ 
Tanaka, J. W., Kiefer, M., \& Bukach, C. M. (2004). A holistic account of the ownrace effect in face recognition: evidence from a cross-cultural study. Cognition, 93(1), B1-B9.

Tullis, J. G., \& Benjamin, A. S. (2011). On the effectiveness of self-paced learning. Journal of Memory and Language, 64(2), 109-118. doi: 10.1016/j.jml.2010.11.002

Tullis, J. G., Benjamin, A. S., \& Liu, X. (2014). Self-pacing study of faces of different races: metacognitive control over study does not eliminate the cross-race recognition effect. Memory and Cognition, 42(6), 863-875. doi: 10.3758/s13421-014-0409-y

Valentine, T. (1991). A unified account of the effects of distinctiveness, inversion, and race in face recognition. The Quarterly Journal of Experimental Psychology, 43A(2), 161-204.

Valentine, T., \& Bruce, V. (1986). The effect of race, inversion and encoding activity upon face recognition. Acta Psychologica, 61, 259-273.

Wan, L., Crookes, K., Reynolds, K. J., Irons, J. L., \& McKone, E. (2015). A cultural setting where the other-race effect on face recognition has no socialmotivational component and derives entirely from lifetime perceptual experience. Cognition, 144, 91-115. doi: 10.1016/j.cognition.2015.07.011

Yardley, L., McDermott, L., Pisarski, S., Duchaine, B. C., \& Nakayama, K. (2008). Psychosocial consequences of developmental prosopagnosia: A problem of recognition. Journal of Psychosomatic Research, 65, 445-451.

Young, S. G., Bernstein, M. J., \& Hugenberg, K. (2010). When do own-group biases in face recognition occur? Encoding versus post-encoding. Social Cognition, 28(2), 240-250. 
Young, S. G., \& Hugenberg, K. (2012). Individuation motivation and face experience can operate jointly to produce the own-race bias. Social Psychological and Personality Science, 3(1), 80-87.

Zhao, M., Hayward, W. G., \& Bülthoff, I. (2014). Holistic processing, contact, and the other-race effect in face recognition. Vision Research, 105, 61-69. doi: 10.1016/j.visres.2014.09.006 